use of Rituximab, AntiTNFs and Tocilizumab has been described, presenting a good clinical response and with few adverse effects, as has been observed in our patients. Disclosure of Interests: None declared

DOI: 10.1136/annrheumdis-2020-eular.6074

\section{AB0504 THE DIFFERENCES OF IMMUNE CHARACTERISTICS AND ITS RELATIONSHIP WITH DISEASE ACTIVITY IN LARGE VESSEL VASCULITIS PATIENTS}

A. Maimaitiyakefu ${ }^{1}$, L. Wu ${ }^{1} .{ }^{1}$ Department of Rheumatology and Immunology in People's Hospital of Xinjiang Uygur Autonomous Region, China, Urumqi, Xinjiang, China

Background: Large vessel vasculitis(LVV) is a rare refractory disease, giant cell arteritis (GCA) and Takayasu arteritis (TAK) constitute different types of primary LVV. Despite considerable discussion on the potential differences between GCA and TAK, the immunological difference and its relationship to disease activity remains unclear. Objectives: To investigate the differences of peripheral blood immuno-phenotype between TAK and GCA, and to analyze the correlation between immune metabolism level and disease activity in TAK.

Methods: All patients with TAK and GCA who come to our department from January 2010 to January 2020 were included, all of them met the classification criteria of American college of rheumatology.General information, clinical manifestations, laboratory index, artery involvement and therapeutic schedule were collected.According to the National Institutes of Health criteria, there were 29 active and 23 inactive TAK patients. Results:

[1] Baseline characteristics, treatment,involvement artery in LVV patients are summarized in Table 1.

Table 1. Clinical characteristics of patients with LVV

\begin{tabular}{lcc}
\hline Variable & TAK,n=52 & GCA,n=4 \\
\hline Age, $\mathrm{X} \pm \mathrm{S}$ & $29 \pm 13$ & $64 \pm 7$ \\
Female,n(\%) & $45(86.6)$ & $1(25)$ \\
Constitutional symptoms,n(\%) & $23(43.4)$ & $3(75)$ \\
Cephalic symptoms,n(\%) & $33(62.3)$ & $2(50)$ \\
Peripheral limb ischemia,n(\%) & $22(41.5)$ & - \\
Ocular signs,n(\%) & $3(5.7)$ & $2(50)$ \\
Stroke,n(\%) & $2(3.8)$ & - \\
GC,n(\%) & $40(76.9)$ & $3(75)$ \\
GC dose $>7.5 m g / d a y, n(\%)$ & $36(90)$ & $2(66.7)$ \\
Surgical intervention,n(\%) & $7(13.2)$ & - \\
Coronary artery,n(\%) & $3(5.7)$ & - \\
Common carotid artery,n(\%) & $31(58.5)$ & - \\
Subclavian artery,n(\%) & $23(43.4)$ & - \\
thoracic aorta,n(\%) & $6(11.3)$ & - \\
abdominal aorta,n(\%) & $13(24.5)$ & - \\
\hline
\end{tabular}

GC Glucocorticoids,bDMARDS biologic DMARDs

(2) Compared with GCA patients, TAK patients were younger $(29 \pm 13 v s 64 \pm 7, \mathrm{P}=0.015)$, with higher peripheral blood lymphocyte count of CD8+T cells and lower CD4/CD8(Table 2).

Table 2. Comparison of peripheral immune cells, humoral immunity among TAK and GCA, active and inactive TAK

\begin{tabular}{|c|c|c|c|c|c|c|}
\hline Variable & $\mathrm{TAK}, \mathrm{n}=52$ & $\mathrm{GCA}, \mathrm{n}=4$ & $\begin{array}{c}p \\
\text { value }\end{array}$ & $\begin{array}{c}\text { inactive } \\
\text { TAK,n=23 }\end{array}$ & $\begin{array}{c}\text { active } \\
\text { TAK, } n=29\end{array}$ & $\begin{array}{c}p \\
\text { value }\end{array}$ \\
\hline Neutrophils & $5.5 \pm 2.3$ & $5.8 \pm 1.1$ & 0.827 & $4.4 \pm 1.6$ & $6.3 \pm 2.5$ & $0.009^{*}$ \\
\hline Lymphocytes & $2.4 \pm 0.8$ & $1.0 \pm 0.6$ & $0.010^{*}$ & $2.3 \pm 0.7$ & $2.4 \pm 0.9$ & 0.901 \\
\hline Monocytes & $0.5 \pm 0.2$ & $0.7 \pm 0.1$ & 0.218 & $0.5 \pm 0.2$ & $0.6 \pm 0.2$ & 0.431 \\
\hline $\mathrm{CD}^{+} \mathrm{T}$ & $1959 \pm 490$ & $1154 \pm 541$ & 0.189 & $2195 \pm 92$ & $1842 \pm 585$ & 0.467 \\
\hline $\mathrm{CD}^{+}{ }^{+} \mathrm{T}$ & $1074 \pm 305$ & $758 \pm 303$ & 0.381 & $1250 \pm 198$ & $987 \pm 333$ & 0.376 \\
\hline $\mathrm{CD}^{+} \mathrm{T}$ & $859 \pm 212$ & $297 \pm 87$ & $0.048^{*}$ & $945 \pm 41$ & $815 \pm 258$ & 0.542 \\
\hline $\mathrm{CD}^{+} \mathrm{CD} 8^{+}$ & $42 \pm 27$ & $12 \pm 7$ & 0.344 & $63 \pm 2$ & $32 \pm 28$ & 0.229 \\
\hline CD4-CD8- & $90 \pm 75$ & $91 \pm 63$ & 0.999 & $97 \pm 74$ & $87 \pm 68$ & 0.91 \\
\hline $\mathrm{CD} 19^{+} \mathrm{B}$ & $273 \pm 97$ & $191 \pm 94$ & 0.470 & $202 \pm 106$ & $309 \pm 84$ & 0.242 \\
\hline $\mathrm{CD}_{16}{ }^{+} \mathrm{CD} 56^{+}$ & $135 \pm 72$ & $104 \pm 67$ & 0.709 & $112 \pm 63$ & $146 \pm 83$ & 0.639 \\
\hline CD4/CD8 & $1.25 \pm 0.15$ & $2.55 \pm 0.51$ & $0.001^{*}$ & $1.33 \pm 0.26$ & $1.21 \pm 0.10$ & 0.440 \\
\hline $\lg G$ & $11.7 \pm 3.7$ & $8.3 \pm 0.5$ & 0.124 & $10.3 \pm 3.4$ & $12.9 \pm 3.6$ & 0.025 \\
\hline $\lg A$ & $2.6 \pm 1.3$ & $2.3 \pm 0.8$ & 0.724 & $2.1 \pm 1.3$ & $3.0 \pm 1.1$ & 0.02 \\
\hline $\operatorname{lgM}$ & $1.7 \pm 0.9$ & $0.8 \pm 0.2$ & 0.118 & $1.5 \pm 0.8$ & $1.8 \pm 0.9$ & 0.202 \\
\hline $\mathrm{K}$ light chain & $9.5 \pm 3.9$ & $6.6 \pm 0.3$ & 0.222 & $8.1 \pm 3.3$ & $10.8 \pm 4.0$ & $0.033^{*}$ \\
\hline$\lambda$ light chain & $5.2 \pm 1.9$ & $3.9 \pm 0.3$ & 0.260 & $4.7 \pm 1.9$ & $5.8 \pm 1.9$ & 0.086 \\
\hline
\end{tabular}

"P $<0.05$

(3)The levels of neutrophils, monocytes, IgG, IgA and $K$ light chain active TAK were higer than inactive TAK, with lower lymphocyte level (Table 2). Further correlation analysis showed that the levels of neutrophils, IgG, IgA and $K$ light chain were positively correlated with disease activity $(r=0.434, P=0.005 ; r=0.380, P=0.019 ; r=0.452, P=0.004 ; r=0.380, P=0.022)$.
Conclusion: Compared with GCA patients, TAK patients had higer level of CD8 ${ }^{+} \mathrm{T}$ lymphocytes, consistenting with previous studies.In addition, TAK patients had persistent immune cell involvement, while humoral immunity is related to disease activity.Deeper studies may be required about the role of immune profile in in large vessel vasculitis.

References:

[1] Miyabe $\mathrm{C}$, Miyabe $\mathrm{Y}$, et al. An expanded population of pathogenic regulatory T-cells in giant cell arteritis is abrogated by IL-6 blockade therapy. Ann Rheum Dis. 2017;76:898-905.

[2] Kermani TA, Crowson CS, Muratore F, Schmidt J, Matteson EL, Warrington KJ. Extra-cranial giant cell arteritis and Takayasu arteritis: how similar are they? Semin Arthritis Rheum. 2015;44:724-8.

Disclosure of Interests: None declared

DOI: 10.1136/annrheumdis-2020-eular. 4126

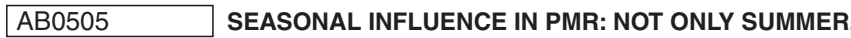 BUT WINTER IS COMING TOO}

D. Marsman ${ }^{1}$, N. Den Broeder ${ }^{2}$, F. Van den Hoogen ${ }^{2}$, A. Den Broeder ${ }^{2}$, A. Van der Maas ${ }^{3} .{ }^{1}$ Sint Maartenskliniek, Rheumatology, Ubbergen, Netherlands; ${ }^{1}$ Sint Maartenskliniek, Rheumatology, Ubbergen, Netherlands; ${ }^{3}$ Sint Maartenskliniek, Ubbergen, Netherlands

Background: The cause for polymyalgia rheumatica (PMR) is currently unknown. Disease onset may be triggered by a combination of genetic predisposition and environmental factors such as infection. ${ }^{1}$ In different regions of Denmark a simultaneous peak incidence of giant cell arthritis and PMR occurred together with epidemics of Mycoplasma pneumoniae, Chlamydophila pneumoniae and Parvovirus B19. ${ }^{1}$ A seasonal epidemics pattern for PMR would be supporting evidence for an infectious cause. ${ }^{1}$ However, the current evidence of seasonal effect on the occurrence and disease severity of PMR is limited and show conflicting results. ${ }^{2,3}$

Objectives: To evaluate whether there is a seasonal effect on the risk of developing PMR in the Netherlands

Methods: We retrospectively collected data on patient-, disease,- and treatment characteristics from newly diagnosed PMR patients (clinical diagnosis) who visited our outpatient clinic during April 2012 and September 2017. Exclusion criteria was other concomitant inflammatory rheumatic disease. Based on the onset of PMR (start symptoms, not time of diagnosis) patients were grouped per month. Descriptive statistics were used [mean (SD), median (p25-p75) or $n$ (\%) as appropriate]. The Chi square goodness of fit test was used to determine whether the incidence of onset of symptoms was different between months of the year.

Results: In total 448 patients were included and $55 \%$ were female and mean age was 66 years. Other baseline characteristics are described in table 1. The chi-square goodness of fit test to determine whether there was a peak incidence in months was $p=0.06$. As shown in figure 1 the incidence of onset PMR symptoms is higher in December-January, April through June with a peak in August. The April-June peaks coincides with incidence peaks of Mycoplasma pneumoniae infections and possibly Parvovirus B19 in spring and summer, the December-January peak coincides with Parvovirus B19 infections. ${ }^{4,5}$

Table 1. Baseline characteristics $(n=448)$

Characteristic

Female (\%)

Age, years (SD)

PMR symptoms before diagnosis, weeks (IQR)

Neck pain (\%)

Bilateral shoulder pain

/stiffness (\%)

Bilateral hip pain/stiffness $(\%)$

Morning stiffness $>45 \mathrm{~min}(\%)$

Peripheral arthritis (\%)

Systemic symptoms* $(\%)$

Elevated ESR mm/hour and / or CRP mg/l

ESR mm/hour(IQR)

CRP mg/l (IQR)

$247(55)$

$66(8.6)$

$10(6-16)$

205 (46)

$412(91)$

380 (85)

$233(52)$

$35(8)$

199 (44)

$37(26-51)$

${ }^{*}$ Fever, night sweats, weight loss, anorexia

** ESR $n=428 ;$ CRP $n=396$

Conclusion: No definitive seasonal effect was found on risk of developing PMR although a bimodal seasonal pattern compatible with the proposed respiratory infections is suggested.

References:

[1] González-Gay MA et al.Polymyalgia rheumatica. Lancet. 2017 Oct Oct 7. 
[2] Narváez J et al.JRheumatol. 2000 Apr;27(4):953-7. Lack of association between infection and onset of polymyalgia rheumatica.

[3] F. Perfetto et al. seasonal pattern in the onset of polymyalgia rheumatic. Abstract EULAR 2005

[4] Chen ZR et al. Epidemiology of community-acquired Mycoplasma Pneumoniae among hospitalized Chinese children.Hippokratia. 2013;17(1):20-26.

[5] Enders $M$ et al. Current epidemiological aspects of human parvovirus B19 infection Epidemiol Infect. 2006;135(4):563-569.

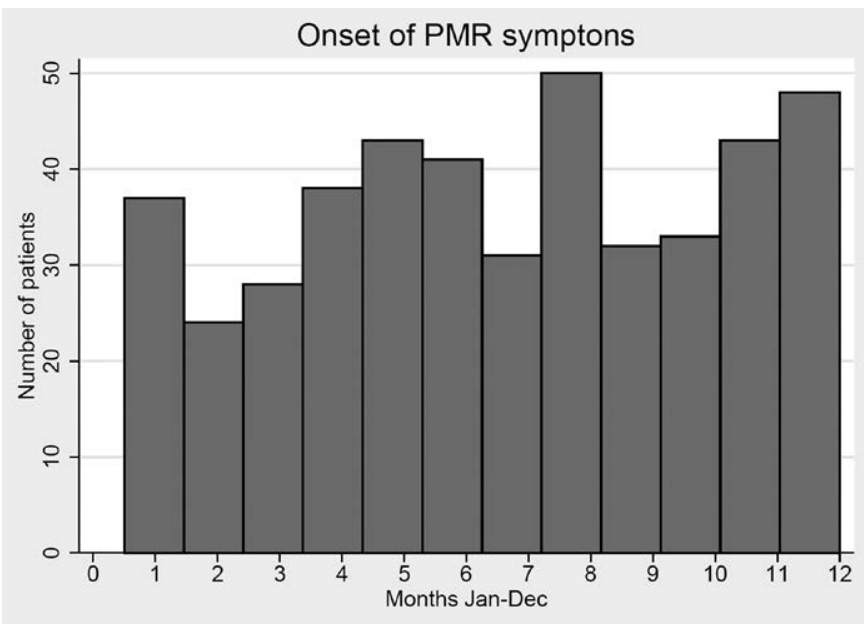

Disclosure of Interests: None declared

DOI: 10.1136/annrheumdis-2020-eular.1717

\section{AB0506 $\quad$ GIANT CELL ARTERITIS: IS ROUTINE CLINICAL PRACTICE COMPREHENSIVE ENOUGH?}

J. Martínez-Barrio $^{1}$, B. Serrano Benavente ${ }^{1}$, T. Del Río Blasco ${ }^{1}$, A. Ariza ${ }^{1}$,

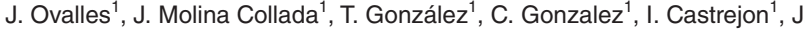
M. Alvaro Gracia'. ' Gregorio Marañón University Hospital, Rheumatology, Madrid, Spain

Background: Recommendations to collect the most relevant information on disease course, treatment and outcomes in giant cell arteritis (GCA) has been proposed by EULAR to facilitate clinical research and to improve clinical care. Objectives: To assess the quality of data collection in routine clinical practice according to EULAR recommendations and to describe baseline and follow-up characteristics of a retrospective cohort of patients with GCA

Table 1. GCA-related signs and symptoms, laboratory and therapeutic data.

\begin{tabular}{lcccc}
\hline Item & $\begin{array}{c}\text { Performed } \\
\text { Baseline }\end{array}$ & $\begin{array}{c}\text { Baseline } \\
\mathrm{n}=39\end{array}$ & $\begin{array}{c}\text { Performed } \\
\text { Follow-up }\end{array}$ & $\begin{array}{c}\text { Follow-up } \\
\mathrm{n}=112\end{array}$ \\
\hline
\end{tabular}

Ocular symptoms

$35 / 39(89.7 \%) \quad 15 / 35(42.9 \%) \quad 91 / 112(81.2 \%) 29 / 91(31.9 \%)$

$\begin{array}{llll}\text { Permanent ocular symptoms } \quad 34 / 39(87 \%) & 9 / 34(26.5 \%) & 92 / 112(82 \%) & 28 / 92(30.4 \%)\end{array}$

Headache

Scalp tenderness

Jaw claudication

Cranial artery abnormality

Constitutional symptoms

PMR

ESR mean (SD)

CRP mean (SD)

Haemoglobin mean (SD)

Peripheral pulses

Large vessel involvement

Glucocorticoids median

(IQR)

Synthetic DMARD

Biological DMARD

Antiplatelet agents

$39(100 \%) \quad 30 / 39(77 \%) \quad 90 / 112(80.4 \%) \quad 13 / 90(14.4 \%)$

$31 / 39(79.5 \%) \quad 9 / 31(29.8 \%) \quad 88 / 112(78.6 \%) \quad 4 / 88(4.5 \%)$

$34 / 39(87 \%) \quad 19 / 34(55.85) \quad 91 / 112(81.2 \%) \quad 6 / 91(6.6 \%)$ $27 / 39(69.2 \%) \quad 17 / 27(63 \%) \quad 69 / 112(61.6 \%) \quad 3 / 69(4.3 \%)$ $35 / 39(89.7 \%)$ 19/35 (54.3\%) 90/112 (80.4\%) $11 / 90(12.2 \%)$ $35 / 39(89.7 \%) \quad 18 / 35(51.4 \%) \quad 92 / 112(82 \%) \quad 9 / 92(9.8 \%)$ $33 / 39(84.6 \%) \quad 58.7(32.1) \quad 83 / 112(74 \%) \quad 14.6(18.8)$ $31 / 39(79.5 \%) \quad 8.4(7.9) \quad 70 / 112(62.5 \%) \quad 1.3(3.3)$ $38 / 39(97.4 \%) \quad 12.0(1.7) \quad 90 / 112(80.4 \%) \quad 12.9(1.5)$ $9 / 39(8 \%) \quad 3 / 9(33.3 \%) \quad 5 / 112(4.5 \%) \quad 2 / 5(40 \%)$ $8 / 39(20.5 \%) \quad 5 / 8(62.5 \%) \quad 7 / 112(6.25 \%) \quad 3 / 7(42.8 \%)$ $39(100 \%) \quad 102.5(50-250) \quad 112(100 \%) \quad 10.0(5-15)$

39 (100\%) $\quad 8 / 39(20.5 \%) \quad 111 / 112(99 \%) \quad 17 / 39(43.6 \%)$ $39(100 \%) \quad 0 / 39(0 \%) \quad 111 / 112(99 \%) \quad 3 / 39(7.7 \%)$ $39(100 \%) \quad 6 / 39(15.4 \%) \quad 110 / 112(98 \%) \quad 25 / 110$

(22.7\%)

PMR: polymyalgia rheumatica, ESR: erythrocyte sedimentation rate, CRP: C-reactive protein, SD: standard deviation, IQR: interquartile range, DMARD: disease modifying antirheumatic drugs
Methods: We reviewed medical records of patients diagnosed with GCA in a tertiary academic center between 2004-2018. We included patients with available data at diagnosis and one year of follow-up. Data extraction included: demographics, diagnosis, GCA-related signs and symptoms, laboratory, imaging modalities, comorbidities and treatment. Data in the chart was then compared with the core set of parameters proposed for GCA registries and databases by EULAR. Major relapse, according to the EULAR 2018 definition, was independently assessed by two rheumatologists.

Results: 58 patients were identified, 39 met predefined inclusion criteria with 151 visits during first-year follow-up. Headache (100\%; $80.4 \%)$, ocular symptoms (89.7\%; 81.2\%), constitutional symptoms (89.7\%; $80.4 \%)$, polymyalgia rheumatica $(89.7 \% ; 82 \%)$ and jaw claudication $(87 \% ; 81.2 \%)$ were the most frequently collected items at baseline and follow-up. Weight and height $(2.6 \%$; $2.6 \%)$, peripheral pulses $(8 \% ; 4.5 \%)$, smoking status $(41 \% ; 21 \%)$, and blood pressure $(61.5 \% ; 4.5 \%)$ were the less frequently collected. Most patients lacked differential pressure measurement. Myocardial infarction, malignancy, serious infections, arterial hypertension, diabetes and osteoporosis were collected in every patient $(39,100 \%)$. Only 2 mayor relapses were identified $(5 \%)$ Two (2) patients died during the one-year follow-up period. Table 1 provides information on GCA-related signs and symptoms, laboratory and therapeutic data.

Conclusion: Although data collection in routine care is usually comprehensive enough according to EULAR proposed data set, key components in physical exam mostly those aiming to detect large vessel involvement, should be addressed more carefully.

References:

[1] Ehlers L, et al. Ann Rheum Dis. 2019;78(9):1160-6.

[2] Hellmich B, et al. Ann Rheum Dis. 2019;1-12.

Disclosure of Interests: Julia Martínez-Barrio Consultant of: UCB Pharma, Belén Serrano Benavente: None declared, Tamara Del Río Blasco: None declared, Alfonso Ariza: None declared, Juan Ovalles: None declared, Juan Molina Collada: None declared, Teresa González: None declared, Carlos Gonzalez Consultant of: Gilead, Janssen, Novartis, Speakers bureau: Abbvie, Celgene, Gilead, Janssen, Novartis, Pfizer, Roche, Isabel Castrejon: None declared Jose Maria Alvaro Gracia: None declared DOI: 10.1136/annrheumdis-2020-eular.4852

\section{AB0507 INVASIVE ASPERGILLOSIS IN ADULT RHEUMATOLOGICAL PATIENTS IN SAINT PETERSBURG, RUSSIA.}

V. Mazurov ${ }^{1}$, O. Shadrivova ${ }^{1}$, M. Shostak ${ }^{1}$, L. Martynova ${ }^{1}$, M. Tonkoshkur ${ }^{1}$, N. Klimko ${ }^{1}{ }^{1}$ North-Western State Medical University named after I.I. Mechnikov, Saint Petersburg, Russian Federation

Background: Invasive aspergillosis (IA) is a severe opportunistic infection that is not well understood in rheumatological patients.

Objectives: To study risk factors, etiology, clinical manifestations and results of treatment of IA in adult rheumatological patients.

Methods: Retrospective analysis of 830 patients (1998-2019) with "proven" and "probable" IA (EORTC / MSG, 2019), adults - 699 (84\%). The main group included $18(3 \%)$ adult rheumatological patients with IA, a control group included $610(87 \%)$ adult hematological patients. Rheumatological patients were older, the average age was 59 years $(21-75)$ vs 45 years $(18-79), p=0.005$, and among them there were more women $-56 \%$ vs $42 \%, p=0.01$.

Results: In rheumatological patients with IA, underlying diseases were ANCA-associated vasculitis (28\%), granulomatosis with polyangiitis $(22 \%)$, periarteritis (11\%), systemic lupus erythematosus $(22 \%)$, rheumatic heart disease $(11 \%)$ and ankylosing spondylitis (6\%). In the control group, underlying diseases were acute leukemia (45\%), lymphomas (34\%), chronic leukemia $(9 \%)$, multiple myeloma $(7 \%)$, myelodysplastic syndrome $(3 \%)$, and other hematological diseases (2\%)

The main risk factors for IA development in rheumatological patients were: systemic steroids use ( $89 \%$ vs $69 \%$ ), prolonged lymphocytopenia ( $76 \%$ vs $65 \%$, median - 14 vs 12 days), treatment in ICU ( $44 \%$ vs $18 \%, p=0.01)$, acute or chronic renal failure (39\% vs $1 \%, p=0.0008)$ and immunosuppressive therapy ( $28 \%$ vs $25 \%$ ). Severe neutropenia was noted significantly less frequently ( $18 \%$ vs $83 \%, p=0.0001)$. Additional risk factors were decompensated diabetes mellitus $(17 \%$ vs $2 \%, p=0.004)$, previous surgery $(17 \%$ vs $1 \%, p=0.001)$ and organ transplantation ( $6 \%$ vs $0 \%)$. In rheumatological patients, lung $(83 \%$ vs $98 \%, p=$ $0.0001)$ and $\geq 2$ organs ( $6 \%$ vs $8 \%$ ) involvement were less common. Heart ( $11 \%$ vs $0 \%$ ), sinuses $(6 \%$ vs $5 \%$ ) and central nervous system (6\% vs $4 \%$ ) involvement more often developed. In rheumatological patients, respiratory failure $(61$ vs $37 \%, p=0.03)$, hemoptysis $(28 \%$ vs $7 \%, p=0.0001)$ and chest pain $(17 \%$ vs $7 \%, p=0,04)$ were noted more often, less often - fever $\geq 38^{\circ} \mathrm{C}(67 \%$ vs $85 \%$, 